\title{
ESTUDIO DE LAS TEORÍAS IMPLÍCITAS DE LA EDUCACIÓN FÍSICA EN LA FORMACIÓN INICIAL DE LOS MAESTROS EN LAS DIFERENTES ESPECIALIDADES DE LA FACULTAD DE CIENCIAS DE LA EDUCACIÓN DE LA UNIVERSIDAD DE GRANADA. DIFERENCIAS EN FUNCIÓN DEL GÉNERO
}

\section{Autores}

DELGADONOGUERA, MIGUEL ÁNGEL ZURITA MOLINA, FÉLIX UNIVERSIDAD DE GRANADA

\section{Resumen}

Este artículo pretende conocer cuáles son y cómo evolucionan a lo largo de su formación inicial las ideas, las concepciones previas, las teorías implícitas que traen o tienen nuestros alumnos y alumnas, futuros maestros y maestras, acerca de la Educación Física.

El género como, como señala Fernández (1995) es una realidad compleja fundamentalmente psicosocial, que se asienta en un comienzo, en la variable sexo y que interactúa continuamente con ella a lo largo de todo el ciclo vital. ¿En qué medida ser mujer o varón influye en la manera de ver y concebir la EF?. Esta influencia ise puede trasmitir al alumnado?, sería un paso más a estudiar.

Nuestra hipótesis de partida es que los varones, futuros maestros, muestran un interés mayor por la EF como rendimiento deportivo y las mujeres, futuras maestras, por la EF como materia educativa y como expresión. En el resto de teorías no pensamos que se aprecien diferencias importantes.

Las teorías implícitas de la Educación Física se generan a través de la experiencia vivida, en relación con lo conceptual (teoría), la práctica, la salud y sus implicaciones axiológicas, afectivas y sociales. Las teorías estudiadas son: Teoría EF Salud, Teoría EF Rendimiento, Teoría EF Recreación, Teoría EF Expresión y Teoría EF Educación (integral).

Podemos constatar en las conclusiones que entre los futuros maestros hay diferencias en función del género y ello nos lleva a considerar que éste es un elemento mediador en el proceso de enseñanza-aprendizaje y en las teorías implícitas. Los varones valoran, en general, más que las mujeres las distintas concepciones o teorías de la EF. Los varones valoran más la EF como recreación no cumpliéndose, en este caso, nuestra hipótesis. Las mujeres valoran más la EF como salud. La teoría implícita menos valorada es la EF como rendimiento tanto en las mujeres como en los varones. Entre las especialidades estudiadas, los valores más altos se dan en la especialidad de EF tanto en los varones como en las mujeres.

\section{Introducción}

Siguiendo el mismo estudio sobre las teorías implícitas de la Educación Física (EF) en la formación inicial de los maestros en las diferentes especialidades, (Delgado y Zurita, 2002), hemos querido conocer si existen opiniones diferenciadas en función del género. ¿Es el géne- ro un factor que marca diferencias en las concepciones acerca de la EF escolar?, ¿Están condicionadas estas diferencias en función de las diferentes especialidades? y ¿Existen diferencias en la evolución a lo largo de los estudios dentro de la especialidad de EF?. 
Como indicábamos en el estudio anterior, los estudios sobre la enseñanza de la EF (Wittrock, 1989) han estado centrados en la figura del profesor de EF y en la preparación profesional de los futuros enseñantes de la Educación Física durante la formación inicial (Delgado, 1997) centrándose especialmente en la formación de competencias docentes. El interés científico por la formación inicial del profesorado hoy también se interesa por el pensamiento del profesorado de Educación Física (EF) respecto a las teorías implícitas. Martínez (1994) y Delgado, Medina y Viciana (1996).

Entendemos que las teorías implícitas son síntesis dinámicas de experiencias biográficas que se activan por demandas del sistema cognitivo. (Rodríguez, Rodrigo y Marrero, 1993) Éstas son directas, vicarias o simbólicas. En EF se han estudiado en Medina y Delgado (1998), Delgado, Medina y Chillón (2002) y Delgado (2002).

Así nos interesa conocer cuáles son las concepciones previas que tienen los futuros maestros en función del género acerca de la EF y de la propia enseñanza al iniciar sus estudios de formación de profesorado:

Es frecuente que algunos alumnos, futuros maestros y maestras, tengan la idea de que en la clase de EF hay que conseguir que el alumnado se lo pase bien. Tienen una preconcepción de que en la EF lo primordial es lo lúdico, vivencial y recreativo. ¿Esta concepción es más típica de los maestros o de las maestras?

Otros futuros maestros y maestras tienen la idea previa de la clase de EF como un lugar para enseñar deportes y donde lo normal será que aprendan la técnica y la táctica para practicarlos. Centran su pretensión en que cuando termine el curso su alumnado pueda practicar mucho mejor el deporte típico escolar que se realiza en ese centro educativo. ¿Esta concepción está condicionada por el hecho de tratarse de varones o mujeres?

$A$ veces, algunos futuros maestros y maestras tienen una idea muy clara de que en la clase de EF tiene que desarrollar la condición física y lograr que los alumnos sean más fuertes, más ágiles y resistentes; en definitiva, alcanzar el máximo rendimiento físico. ¿Esta concepción es más típica en los maestros o en las maestras?

Para algunos profesores o futuros maestros las funciones de la EF son percibidas de manera muy diferente, en función de sus experiencias previas, expectativas acerca de la materia, importancia que le conceden a esta asignatura e ideas personales. ¿Puede influir también el género?.

Según el cuadro $N^{\circ} 1$ podemos observar las funciones y sus relaciones con las diferentes concepciones educativas acerca de la EF.
Este trabajo pretende conocer cuáles son y cómo evolucionan a lo largo de su formación inicial las ideas, las concepciones previas, las teorías implícitas que traen o tienen nuestros alumnos y alumnas, futuros maestros y maestras, acerca de la Educación Física. El procedimiento es de corte transversal y es nuestra intención continuar el estudio de forma longitudinal durante los tres años que duran los estudios del maestro con especialidad en EF.

Entendemos el género como "una realidad compleja fundamentalmente psicosocial, que se asienta en un comienzo, en la variable sexo y que interactúa continuamente con ella a lo largo de todo el ciclo vital. El estudio de los roles, estereotipos, masculinidad, feminidad, etc ... se encuadraría dentro de esta realidad de género" (Fernández, 1995).

Aunque en nuestro estudio la comparación se realiza entre los varones y las mujeres (como variable sexo, la hacemos extensiva al género, por las razones antes manifestadas en la definición que hemos recogido previamente). El objeto de estudio, por tanto, en este trabajo son las teorías implícitas en función del género.

La EF y el género es un tópico de investigación muy importante. Diferentes autores han realizado estudios sobre este tema: Vázquez, Fernández, et alii, 2000; Bonal, 1997; Fernández, 1995; Bonilla y Martínez, 1992; Subirats y Tome, 1992; McBride, 1990; Vázquez y Álvarez, 1990; Granleese, Turner, Trew, 1989 y Griffin, 1985.

En los estudios de estos autores, resumidamente, se recoge que:

Los chicos participan más en los deportes y juegos colectivos privando a las chicas acceder a la práctica.

Los chicos y las chicas interactúan según modelos tradicionales estereotipados.

Las formas competitivas masculinas dominan todavía la concepción de la EF y los criterios de evaluación. (Tomado de Vázquez, Fernández, et alii, 2000.) En el estudio que realizan en lo que a nosotros nos interesa reflejan que:

a) Tanto la práctica de la actividad física en el centro como fuera del mismo es más alta en los chicos que en las chicas y tanto por número de deportes practicados como por la frecuencia de dicha práctica.

b) Las prácticas de las actividades físicas está asociada, en gran medida, a los estereotipos de género: así las chicas practican más deportes de adscripción femenina (aeróbic, baloncesto, gimnasia rítmica, baile, natación ...); en cambio los chicos se concentran más en fútbol, ciclismo y balonmano.

Funciones
Conocimiento
Anatómico - funcional.
Estética y expresiva.
Comunicativa y de relación.
Higiénica.
Agonística.
Catártica y hedonista.
Compensación.

Integradora.
Rendimiento físico - motriz.
Expresiva.
Recreativa.
Salud física.
Rendimiento.
Socio - recreativa.
Salud psicológica y física.

Cuadro $N^{o} 1$. Relaciones entre funciones $y$ concepciones de la Educación Física. 
c) Aunque las chicas tienden a romper el estereotipo femenino clásico, sin embargo, siguen implicándose menos en la clase de EF y tienen un autoconcepto más bajo que los alumnos respecto a su interés y competencia motriz.

Aunque en nuestro estudio sólo vamos a tratar acerca de las teorías que se constatan en función del género, nuestra hipótesis de partida se manifiesta en que los varones, futuros maestros, muestran un interés mayor por la EF como rendimiento deportivo y las mujeres, futuras maestras, por la EF como materia educativa y como expresión. En el resto de teorías no pensamos que se aprecien diferencias importantes.

\section{Las teorias implicitas de la educación física}

Las teorías implícitas sobre la EF por parte de alumnado, futuro maestro o maestra, suponen una síntesis de lo que se conoce y piensa sobre esta materia educativa y constituye una abanico de concepciones alternativas que el alumnado es capaz de reconocer y discriminar acerca de la EF.

Las teorías implícitas de la Educación Física se generan a través de la experiencia vivida, en relación con lo conceptual (teoría), la práctica, la salud y sus implicaciones axiológicas, afectivas y sociales.

Conocer cuáles son las teorías implícitas de la EF, detectar las preconcepciones, antes de empezar la formación es importante para poder actuar con mayor eficacia, para hacer consciente al propio alumnado de la estructura mental y cultural que tienen acerca de la materia.

En este sentido también es de interés conocer el impacto que produce el curriculum formativo de la EF en el conocimiento teórico y científico de la materia, en lo que se considera importante como contenido y práctica de la EF (bloques de contenidos prácticos y procedimientos), y en las actitudes y valores adquiridos sobre la EF y lo corporal.

Las teorías implícitas del maestro y maestra analizadas son las mismas que las estudiadas por Delgado y Zurita (2002) Estas teorías surgieron tras una revisión y análisis documental de textos, después de haber realizado un grupo de discusión con estudiantes de EF y tras una primera revisión de expertos universitarios, etc., siguiendo el procedimiento de Marrero (1993):

I. TEORÍA SALUD: Considera esencial la Educación Física realizada como medio de promoción de la salud. Concede a la EF un valor sobre todo de tipo rehabilitador y de recuperación de tipo físico y de apoyo a dificultades en el aprendizaje escolar. Enfoca la Educación Física también como un medio de prevenir enfermedades en el alumnado.

II. TEORÍA RENDIMIENTO: Pretende que la EF fundamentalmente se dedica a poner fuerte a los individuos. Considera que la Educación Física es esencial- mente el rendimiento físico y deportivo. Predomina el producto frente al proceso. Le concede a la Educación Física un valor de utilidad para realizar actividades de la vida diaria. Se centra fundamentalmente en el deporte como contenido esencial de la Educación Física.

III. TEORÍA RECREATIVA: Interpreta la Educación Física que se realiza en las clases como un medio de entretenimiento y diversión. En EF lo importante es el proceso y pasárselo bien.

IV. TEORÍA EXPRESIVA: Entiende la Educación Física como un medio de mejorar su imagen corporal que te permite moldear tu cuerpo. El dominio esencial de la EF es la expresión y comunicación corporal.

V. TEORÍA EDUCATIVA: Le concede a la Educación Física un valor formativo y educativo relacionado con la educación del individuo. Considera que la Educación Física desarrolla de forma completa al sujeto. Adopta un enfoque filosófico de la Educación Física. Plantea la Educación Física como una materia escolar que hay que realizar. La Educación Física tiene un valor cultural y éste varía en función del valor que le da cada sociedad. La Educación Física tiene su importancia porque te permite relacionarte socialmente y facilita las relaciones entre el alumnado.

Estas concepciones o teorías implícitas cestán condicionadas por el género de los futuros maestros y maestras de EF? Si el género es una variable que interactúa y actúa como mediador habrá que pensar que en función del género del maestro o maestra, éste puede influir en el alumnado a través de un enfoque diferente en su enseñanza de la EF.

\section{Aplicación del cuestionario a los futuros maestros}

Para la aplicación del cuestionario seguimos el procedimiento metodológico planteado por Marrero, (1993).

\section{Sujetos}

Se aplicó el cuestionario TIEFP (Teorías Implícitas Educación Física Profesorado) en la Facultad de Ciencias de la Educación de la Universidad de Granada a un total de 219 alumnos, de los cuales 97 son mujeres $(44,29 \%)$ y 122 son hombres $(55,71 \%)$, según dos criterios de agrupación:

a) Los primeros cursos de magisterio de las especialidades de Educación Física (EF), Primaria (EPO), Música (EMU) y Lengua Extranjera (LEX).

Entre los alumnos que rellenaron el cuestionario predominan los varones en EF y Música y las mujeres en Primaria y Lengua Extranjera. Ver tabla $N^{0} 1$. 


\begin{tabular}{|c|c|c|c|c|c|c|c|c|c|c|c|}
\hline \multicolumn{3}{|c|}{$\begin{array}{l}1^{\circ} \text { Magisterio } \\
\text { Educación Física }\end{array}$} & \multicolumn{3}{|c|}{$\begin{array}{l}1^{\circ} \text { Magisterio } \\
\text { Primaria }\end{array}$} & \multicolumn{3}{|c|}{$\begin{array}{l}\text { 10 Magisterio } \\
\text { Música }\end{array}$} & \multicolumn{3}{|c|}{$\begin{array}{c}1^{\circ} \text { Magisterio } \\
\text { Lengua Extranjera }\end{array}$} \\
\hline Mujeres & Hombres & $T$ & Mujeres & Hombres & T & Mujeres & Hombres & T & Mujeres & Hombres & $T$ \\
\hline 19 & 42 & 61 & 28 & 10 & 38 & 8 & 19 & 27 & 21 & 7 & 28 \\
\hline $31,15 \%$ & $68,85 \%$ & & $73,68 \%$ & $26,32 \%$ & & $29,63 \%$ & $70,37 \%$ & & $75 \%$ & $25 \%$ & \\
\hline
\end{tabular}

Tabla $N^{o} 1$. Sujetos de los primeros cursos de magisterio de las especialidades de Educación Física, Primaria, Música y Lengua Extranjera.

b) A todos los cursos de la especialidad de Educación Física.

En todos los cursos hay aproximadamente dos tercios de varones y un tercio de mujeres. Ver tabla $N^{\circ} 2$.
Para este estudio, como se ha indicado antes, se calcularon la tipicidades y polaridades de los sujetos del estudio.

a) Indice de tipicidad

\begin{tabular}{|c|c|c|c|c|c|c|c|c|}
\hline \multicolumn{3}{|c|}{$\begin{array}{l}1^{\circ} \text { Magisterio } \\
\text { Educación Física }\end{array}$} & \multicolumn{3}{|c|}{$\begin{array}{l}2^{\circ} \text { Magisterio } \\
\text { Educación Física }\end{array}$} & \multicolumn{3}{|c|}{$\begin{array}{c}3^{\circ} \text { Magisterio } \\
\text { Educación Física }\end{array}$} \\
\hline Mujeres & Hombres & $T$ & Mujeres & Hombres & $T$ & Mujeres & Hombres & $\mathrm{T}$ \\
\hline 19 & 42 & 61 & 10 & 21 & 31 & 11 & 23 & 34 \\
\hline $31,15 \%$ & $68,85 \%$ & & $32,26 \%$ & $67,74 \%$ & & $32,35 \%$ & $67,65 \%$ & \\
\hline
\end{tabular}

Tabla $N^{o}$ 2. Sujetos de los cursos de magisterio de la especialidad de Educación Física.

Se les pasó el cuestionario durante el mes de junio del año 2002. Se eligió, al azar, un grupo de clase de alumnos.

Se eliminaron un total de quince cuestionarios por varias causas: No completar las cincuenta preguntas y rellenar todas las preguntas con el mismo valor (máximo, mínimo o intermedio).

\section{Cuestionario sobre la Educación Física. Profesorado.}

Este cuestionario normativo TIEFP pretende averiguar la estructura de conocimiento de los futuros maestros en el dominio de EF escolar y comprobar si las teorías históricas tienen entidad y están representadas.

El cuestionario tiene 50 preguntas, diez por cada una de las cinco teorías. Los enunciados de las diferentes teorías están entremezclados de forma que no se aprecie en la continuidad de los enunciados de las diferentes teorías y están barajadas de forma aleatoria. Véase anexo 1. Cuestionario sobre la Educación Física. Profesorado. (TIEFP).

Después de la aplicación del cuestionario se calculan los índices de tipicidad y polaridad del sujeto y /o del grupo de clase en cada teoría. Para conocer las correspondencias y poder efectuar los cálculos tenemos que conocer cuáles son los diez enunciados de cada una de las cinco teorías. Ver anexo 2. Correspondencia de los enunciados y las teorías del cuestionario sobre la Educación Física. Profesorado.

Como fase previa se ha calculado la tipicidad y polaridad de los enunciados para conocer los enunciados típicos de cada teoría que se manifestaban en valores altos y al mismo tiempo la polaridad de los enunciados. Delgado y Zurita (2002).
El índice de tipicidad nos informa acerca de la pertenencia de un sujeto / grupo con respecto a una determinada teoría.

\section{b) Indice de polaridad}

El índice de polaridad nos determina en qué medida puede considerarse un sujeto / grupo más o menos exclusivo de una cierta teoría.

Los resultados de la tipicidad y polaridad nos muestran las teorías más significativas así como la pertenencia o no de los sujetos o del grupo de clase a esas teorías.

\section{Análisis de los resultados}

Los resultados se van a analizar de dos formas:

a) Comparación de las teorías implícitas de la EF del alumnado de primer curso de la Facultad de Ciencias de la Educación de la Universidad de Granada en los estudios de la diplomatura de magisterio según sean varones o mujeres; Se analizarán por separado primero varones y mujeres y luego comparando ambos.

b) Evolución de las teorías implícitas de la EF del alumnado de magisterio, especialidad de EF, a lo largo de los tres años según sean varones o mujeres. Se analizarán por separado primero varones y mujeres y luego comparando ambos.

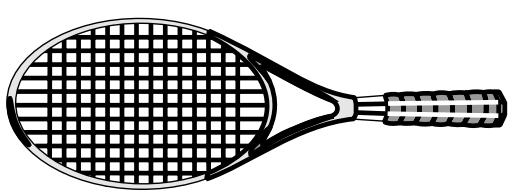


Comparación de las teorías implícitas de la EF del alumnado de primer curso de la Facultad de Ciencias de la Educación en los estudios de la diplomatura de magisterio en función del género. Las especialidades analizadas son: Educación Física, Primaria, Educación Musical y Lengua Extranjera.

Análisis Varones. En su análisis podemos considerar:

$1^{\circ}$. En los varones la valoración de las tipicidades, entre las especialidades, es mayor en el primer curso de Lengua Extranjera donde se valora más la EF como rendimiento, la EF como recreación y la EF como expresión, excepto en el primer curso de EF, que valora más la EF como salud y la EF como educación. Ver tablas $N^{\circ} 3$ y 4.

$2^{\circ}$. La EF recreación en los varones es valorada en primer lugar en todas las especialidades, con excepción del primer curso de Educación Musical que valora la EF como salud.

$3^{\circ}$. La EF rendimiento es valorada en los varones en último lugar en todas las especialidades estudiadas.

$4^{\circ}$. La EF salud en los varones se valora en segundo lugar, excepto en primero de Educación Musical que es la primera valorada. En el resto de las teorías fluctúan de lugar.

$5^{\circ}$. En el promedio total de las valoraciones de las tipicidades en los varones destaca el primer curso de la especialidad de EF, seguido del primer curso de la Lengua Extranjera, Educación Primaria y Educación Musical.

$6^{\circ}$. Las tendencias, antes expresadas de las tipicidades en los varones, coincide con los valores de las polaridades.
Análisis Mujeres. Podemos considerar lo siguiente:

$1^{\circ}$. En las mujeres la valoración de las tipicidades, entre especialidades, es mayor en el primer curso de EF como salud, recreación, expresión y educación, excepto en Primaria con la EF como rendimiento. Ver tablas $N^{0} 5$ y 6.

$2^{\circ}$. En las mujeres la valoración de las tipicidades de la EF como salud es mayor en todas las especialidades, con excepción en primero de EF con la EF como recreación.

$3^{\circ}$. En último lugar se valora a la EF rendimiento en todas las especialidades estudiadas.

$4^{\circ}$. En segundo lugar aparece la EF como recreación en Primaria y Lengua Extranjera.

$5^{\circ}$. En el promedio total de las valoraciones de las tipicidades en las mujeres destaca el primer curso de la especialidad de EF, seguido del primer curso de la Educación Primaria, Lengua Extranjera y Educación Musical.

$6^{\circ}$. Las tendencias, antes expresadas de las tipicidades en las mujeres, coincide con los valores de las polari-

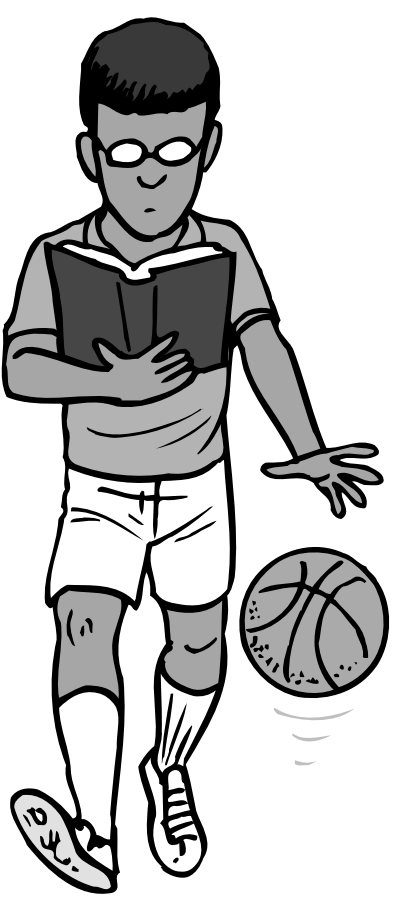
dades.

\begin{tabular}{|l|c|c|c|c|}
\hline Tipicidades & $\begin{array}{c}\mathbf{1}^{\bullet} \text { Magisterio } \\
\text { Educa. Física }\end{array}$ & $\begin{array}{c}\mathbf{1}^{\bullet} \text { Magisterio } \\
\text { Primaria }\end{array}$ & $\begin{array}{c}\mathbf{1}^{\bullet} \text { Magisterio } \\
\text { Educ. Musical }\end{array}$ & $\begin{array}{c}\mathbf{1}^{\circ} \text { Magisterio } \\
\text { L. Extranjera }\end{array}$ \\
\hline Promedio total & 5,06 & 4,96 & 4,15 & 5,03 \\
\hline Tipicidad EF Salud & 5,47 & 5,28 & 4,71 & 5,38 \\
\hline Tipicidad EF Rendimiento & 3,81 & 4,18 & 2,83 & 4,38 \\
\hline Tipicidad EF Recreación & 5,77 & 5,61 & 4,43 & 5,84 \\
\hline Tipicidad EF Expresión & 5,00 & 5,03 & 4,23 & 5,08 \\
\hline Tipicidad EF Educación & 5,23 & 4,70 & 4,55 & 4,47 \\
\hline
\end{tabular}

Tabla $N^{o}$ 3: Tipicidades de las teorías implícitas de la EF del alumnado de primer curso de Magisterio.

\begin{tabular}{|l|c|c|c|c|}
\hline Tipicidades & $\begin{array}{c}1^{\circ} \text { Magisterio } \\
\text { Educa. Física }\end{array}$ & $\begin{array}{c}\mathbf{1}^{\circ} \text { Magisterio } \\
\text { Primaria }\end{array}$ & $\begin{array}{c}\mathbf{1}^{\circ} \text { Magisterio } \\
\text { Educ. Musical }\end{array}$ & $\begin{array}{c}1^{\circ} \text { Magisterio } \\
\text { L. Extranjera }\end{array}$ \\
\hline Promedio total & 5,06 & 4,96 & 4,15 & 5,03 \\
\hline Tipicidad EF Salud & 5,47 & 5,28 & 4,71 & 5,38 \\
\hline Tipicidad EF Rendimiento & 3,81 & 4,18 & 2,83 & 4,38 \\
\hline Tipicidad EF Recreación & 5,77 & 5,61 & 4,43 & 5,84 \\
\hline Tipicidad EF Expresión & 5,00 & 5,03 & 4,23 & 5,08 \\
\hline Tipicidad EF Educación & 5,23 & 4,70 & 4,55 & 4,47 \\
\hline
\end{tabular}

Tabla $N^{o}$ 4: Polaridades de las teorías implícitas de la EF del alumnado de primer curso de Magisterio. Varones. 


\begin{tabular}{|l|c|c|c|c|}
\hline Tipicidades & $\begin{array}{c}\mathbf{1}^{\bullet} \text { Magisterio } \\
\text { Educa. Física }\end{array}$ & $\begin{array}{c}\mathbf{1}^{\bullet} \text { Magisterio } \\
\text { Primaria }\end{array}$ & $\begin{array}{c}\mathbf{1}^{\bullet} \text { Magisterio } \\
\text { Educ. Musical }\end{array}$ & $\begin{array}{c}\mathbf{1}^{\bullet} \text { Magisterio } \\
\text { L. Extranjera }\end{array}$ \\
\hline Promedio total & 4,80 & 4,52 & 4,09 & 4,46 \\
\hline Tipicidad EF Salud & 5,40 & 5,20 & 5,11 & 5,25 \\
\hline Tipicidad EF Rendimiento & 3,19 & 3,65 & 2,95 & 3,64 \\
\hline Tipicidad EF Recreación & 5,52 & 4,99 & 4,29 & 5,10 \\
\hline Tipicidad EF Expresión & 4,75 & 4,51 & 4,41 & 4,38 \\
\hline Tipicidad EF Educación & 5,10 & 4,25 & 3,69 & 3,93 \\
\hline
\end{tabular}

Tabla $N^{o}$ 5: Tipicidades de las teorías implícitas de la EF del alumnado de primer curso de Magisterio. Mujeres.

\begin{tabular}{|l|c|c|c|c|}
\hline Polaridades & $\begin{array}{c}1^{\bullet} \text { Magisterio } \\
\text { Educa. Física }\end{array}$ & $\begin{array}{c}1^{\bullet} \text { Magisterio } \\
\text { Primaria }\end{array}$ & $\begin{array}{c}1^{\bullet} \text { Magisterio } \\
\text { Educ. Musical }\end{array}$ & $\begin{array}{c}1^{\circ} \text { Magisterio } \\
\text { L. Extranjera }\end{array}$ \\
\hline Polaridad EF Salud & 0,11 & 0,12 & 0,18 & 0,14 \\
\hline Polaridad EF Rendimiento & $-0,29$ & $-0,16$ & $-0,20$ & $-0,15$ \\
\hline Polaridad EF Recreación & 0,13 & 0,08 & 0,04 & 0,11 \\
\hline Polaridad EF Expresión & $-0,01$ & 0,00 & 0,06 & $-0,01$ \\
\hline Polaridad EF Educación & 0,06 & $-0,05$ & $-0,07$ & $-0,09$ \\
\hline
\end{tabular}

Tabla $N^{\circ}$ 6: Polaridades de las teorías implícitas de la EF del alumnado de primer curso de Magisterio. Mujeres.

\section{Análisis comparativo Varones y Mujeres}

$1^{\circ}$. Podemos comprobar que las valoraciones más altas (ver tabla $N^{\circ} 7$ ) son la EF como recreación en primer curso de Lengua Extranjera $(5,84)$ en primer curso de EF $(5,77)$, en primer curso de Primaria $(5,61)$.

$2^{\circ}$. En el promedio total de las tipicidades los varones obtienen puntuaciones más altas que las mujeres en todas las especialidades.

$3^{\circ}$. En todas las especialidades las tipicidades de las distintas teorías es mayor en los varones, excepto EF como salud, EF como rendimiento y EF como expresión en la Educación Musical. En el caso de la expresión puede tener su lógica ya que entre la música y la expresión existe relación.
Evolución de las teorías implícitas de la EF del alumnado de magisterio, especialidad de EF, a lo largo de los tres años.

Análisis Varones. En la evolución de los varones a lo largo de las diferentes cursos de la especialidad se aprecian los siguientes hechos destacados:

$1^{\circ}$. En general se mantiene una valoración alta, "bastante de acuerdo", con un promedio de cinco. Ver tablas $N^{\circ} 8$ y 9.

$2^{\circ}$. La evolución más clara se da en la EF como Expresión, partiendo de que los chicos la valoran menos al inicio que al final, con una mejora de 0,56.

$3^{\circ}$. En sentido contrario podemos destacar que la EF como recreación con-0,32. Este hecho se diferencia con respecto a un análisis global sin diferenciar género.

\begin{tabular}{|l|c|c|c|c|c|c|c|c|}
\hline Tipicidades & $\begin{array}{c}1^{\bullet} \mathrm{EF} \\
\text { Varones }\end{array}$ & $\begin{array}{c}1^{\circ} \mathrm{EF} \\
\text { Mujeres }\end{array}$ & $\begin{array}{c}1^{\circ} \text { EPO } \\
\text { Varones }\end{array}$ & $\begin{array}{c}1^{\circ} \text { EPO } \\
\text { Mujeres }\end{array}$ & $\begin{array}{c}1^{\circ} \text { EMU } \\
\text { Varones }\end{array}$ & $\begin{array}{c}1^{\circ} \text { EMU } \\
\text { Mujeres }\end{array}$ & $\begin{array}{c}1^{\circ} \text { LEX } \\
\text { Varones }\end{array}$ & $\begin{array}{c}1^{\bullet} \text { LEX } \\
\text { Mujeres }\end{array}$ \\
\hline Promedio Total & 5,06 & 4,80 & 4,96 & 4,52 & 4,15 & 4,09 & 5,03 & 4,46 \\
\hline Tipicidad EF Salud & 5,47 & 5,40 & 5,28 & 5,20 & 4,71 & 5,11 & 5,38 & 5,25 \\
\hline Tipicidad EF Rendimiento & 3,81 & 3,19 & 4,18 & 3,65 & 2,83 & 2,95 & 4,38 & 3,64 \\
\hline Tipicidad EF Recreación & 5,77 & 5,52 & 5,61 & 4,99 & 4,43 & 4,29 & 5,84 & 5,10 \\
\hline Tipicidad EF Expresión & 5,00 & 4,75 & 5,03 & 4,51 & 4,23 & 4,41 & 5,08 & 4,38 \\
\hline Tipicidad EF Educación & 5,23 & 5,10 & 4,70 & 4,25 & 4,55 & 3,69 & 4,47 & 3,93 \\
\hline
\end{tabular}

Tabla $N^{o}$ 7: Comparación de las tipicidades de las teorías implícitas de la EF del alumnado de primer curso de Magisterio en las especialidades. Varones y Mujeres. 
$4^{\circ}$. La teoría implícita más valorada es en el primer curso de EF con la EF como recreación, en el segundo curso es la EF como salud y en el tercer curso la EF como expresión.

$5^{\circ}$. La peor valorada es al EF como rendimiento.

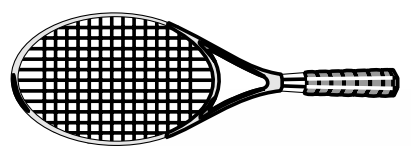

\section{Análisis Mujeres}

$1^{\circ}$. En general, la valoración de las mujeres es algo menor a cinco. Ver tablas $N^{\circ} 10$ y 11.

$2^{\circ}$. Al igual que en los varones, la EF como expresión es la que manifiesta una mayor evolución, con una diferencia de 0,35.

$3^{\circ}$. La teoría que empeora en su valoración más están la EF recreación (-0.69) y la EF salud $(-0,52)$. En este último dato sorprende esta tendencia en las chicas. También empeora la EF rendimiento.

\begin{tabular}{|l|c|c|c|}
\hline Tipicidades & $\begin{array}{c}1^{\bullet} \text { Magisterio } \\
\text { Educación Física }\end{array}$ & $\begin{array}{c}2^{\circ} \\
\text { Educación Física }\end{array}$ & $\begin{array}{c}3^{\circ} \text { Magisterio } \\
\text { Educación Física }\end{array}$ \\
\hline Promedio total & 5,06 & 5,10 & 5,10 \\
\hline Tipicidad EF Salud & 5,47 & 5,59 & 5,25 \\
\hline Tipicidad EF Rendimiento & 3,81 & 3,87 & 3,83 \\
\hline Tipicidad EF Recreación & 5,77 & 5,56 & 5,45 \\
\hline Tipicidad EF Expresión & 5,00 & 5,25 & 5,56 \\
\hline Tipicidad EF Educación & 5,23 & 5,23 & 5,41 \\
\hline
\end{tabular}

Tabla $N^{o}$ 8: Evolución de las tipicidades de las teorías implícitas de la EF del alumnado de Magisterio, especialidad de EF. Varones.

\begin{tabular}{|l|ccc|}
\hline Polaridades & $\begin{array}{c}1^{\circ} \text { Magisterio } \\
\text { Educación Física }\end{array}$ & $\begin{array}{c}2^{\circ} \text { Magisterio } \\
\text { Educación Fisica }\end{array}$ & $\begin{array}{c}3^{\circ} \text { Magisterio } \\
\text { Educación Física }\end{array}$ \\
\hline Polaridad EF Salud & 0,07 & 0,09 & 0,03 \\
\hline Polaridad EF Rendimiento & $-0,22$ & $-0,22$ & $-0,23$ \\
\hline Polaridad EF Recreación & 0,13 & 0,08 & 0,06 \\
\hline Polaridad EF Expresión & $-0,01$ & 0,03 & 0,08 \\
\hline Polaridad EF Educación & 0,03 & 0,02 & 0,06 \\
\hline
\end{tabular}

Tabla $N^{\circ}$ 9: Evolución de las polaridades de las teorías implícitas de la EF del alumnado de Magisterio, especialidad de EF. Varones.

\begin{tabular}{|l|c|c|c|}
\hline Tipicidades & $\begin{array}{c}1^{\circ} \text { Magisterio } \\
\text { Educación Física }\end{array}$ & $\begin{array}{c}2^{\circ} \text { Magisterio } \\
\text { Educación Física }\end{array}$ & $\begin{array}{c}3^{\circ} \text { Magisterio } \\
\text { Educación Física }\end{array}$ \\
\hline Promedio total & 4,80 & 4,43 & 4,56 \\
\hline Tipicidad EF Salud & 5,40 & 5,26 & 4,88 \\
\hline Tipicidad EF Rendimiento & 3,19 & 3,11 & 2,85 \\
\hline Tipicidad EF Recreación & 5,52 & 4,54 & 4,83 \\
\hline Tipicidad EF Expresión & 4,75 & 4,53 & 5,10 \\
\hline Tipicidad EF Educación & 5,10 & 4,68 & 5,12 \\
\hline
\end{tabular}

Tabla $N^{o}$ 10: Evolución de las tipicidades de las teorías implícitas de la EF del alumnado de Magisterio, especialidad de EF. Mujeres.

\begin{tabular}{|l|c|c|c|}
\hline Polaridades & $\begin{array}{c}1^{\circ} \text { Magisterio } \\
\text { Educación Física }\end{array}$ & $\begin{array}{c}2^{\circ} \text { Magisterio } \\
\text { Educación Física }\end{array}$ & $\begin{array}{c}3^{\circ} \text { Magisterio } \\
\text { Educación Física }\end{array}$ \\
\hline Polaridad EF Salud & 0,11 & 0,15 & 0,06 \\
\hline Polaridad EF Rendimiento & $-0,29$ & $-0,23$ & $-0,30$ \\
\hline Polaridad EF Recreación & 0,13 & 0,02 & 0,05 \\
\hline Polaridad EF Expresión & $-0,01$ & 0,02 & 0,10 \\
\hline Polaridad EF Educación & 0,06 & 0,05 & 0,10 \\
\hline
\end{tabular}

Tabla $N^{\circ}$ 11: Evolución de las polaridades de las teorías implícitas de la EF del alumnado de Magisterio, especialidad de EF. Mujeres. 
$4^{\circ}$. En primer curso la teoría más valorada es la recreación, en segundo curso, la EF como salud y en el tercer curso la EF como educación.

$5^{\circ}$. Igual que en los chicos en el último lugar en la valoración aparece la EF rendimiento en todos los cursos. Se da una polarización claramente negativa.

\section{Análisis Varones y Mujeres}

$1^{\circ}$. En la comparación de varones y mujeres siempre y en todos los casos la valoración de los chicos es superior a las de las chicas. Quizás se deba a la tradicional menor importancia que culturalmente ha tenido la EF con respecto al género. Ver tabla $N^{\circ}$ 12. Para entrar en un análisis más cualitativo sería conveniente además de la utilización de este cuestionario o inventario de teorías implícitas, preguntar a los varones y a las mujeres, en base a los resultados obtenidos, las causas o razones que pueden explicar estas diferencias encontradas.
- Conclusión Cuarta: Entre las especialidades, los valores más altos se dan en la especialidad de EF tanto en los varones como en las mujeres.

- Conclusión Quinta: La teoría implícita menos valorada es la EF como rendimiento tanto en las mujeres como en los varones.

Ello nos lleva a considerar que el género es un elemento mediador en el proceso de enseñanza-aprendizaje y en las teorías implícitas. Conviene seguir profundizando en este tópico ya que estamos convencidos que las concepciones previas condicionan nuestras actitudes y valoraciones de la Educación Física y, en muchos casos nuestras propia actuaciones como docentes.

La influencia del profesor o profesora con sus diferentes concepciones puede tener cierta incidencia en el alumnado, lo que nos plantea la conveniencia de su estudio y seguir investigando en este tema.

\begin{tabular}{|c|c|c|c|c|c|c|}
\hline Tipicidades & $\begin{array}{l}1^{\circ} \text { EF } \\
\text { Varones }\end{array}$ & $\begin{array}{l}1^{\circ} \text { E F } \\
\text { Mujeres }\end{array}$ & $\begin{array}{l}2^{\circ} \text { E F } \\
\text { Varones }\end{array}$ & $\begin{array}{l}2^{\circ} \text { E F } \\
\text { Mujeres }\end{array}$ & $\begin{array}{l}2^{\circ} \text { EF } \\
\text { Varones }\end{array}$ & $\begin{array}{l}2^{\circ} \text { E F } \\
\text { Mujeres }\end{array}$ \\
\hline Promedio Total & 5,06 & 4,80 & 5,10 & 4,43 & 5,10 & 4,56 \\
\hline Tipicidad EF Salud & 5,47 & 5,40 & 5,59 & 5,26 & 5,25 & 4,88 \\
\hline Tipicidad EF Rendimiento & 3,81 & 3,19 & 3,87 & 3,11 & 3,83 & 2,85 \\
\hline Tipicidad EF Recreación & 5,77 & 5,52 & 5,56 & 4,54 & 5,45 & 4,83 \\
\hline Tipicidad EF Expresión & 5,00 & 4,75 & 5,25 & 4,53 & 5,56 & 5,10 \\
\hline Tipicidad EF Educación & 5,23 & 5,10 & 5,23 & 4,68 & 5,41 & 5,12 \\
\hline
\end{tabular}

Tabla No 12: Comparación de las tipicidades de las teorías implícitas de la EF del alumnado de Magisterio especialidad en EF en los varones y las mujeres.

\section{Conclusiones}

Podemos constatar que entre los futuros maestros hay diferencia en función del género.

- Conclusión Primera: Los varones valoran, en general, más que las mujeres las distintas concepciones o teorías de la EF.

- Conclusión Segunda: Los varones valoran más la EF como recreación. No se cumple nuestra hipótesis que planteaba que sería la EF como rendimiento la más valorada en los varones.

- Conclusión Tercera: Las mujeres valoran más la EF como salud. Tampoco se cumple nuestra hipótesis que planteaba que sería la EF como expresión la más valorada en las mujeres.

\section{Bibliografía}

BONAL, J. (1997). Cambiar la escuela. La coeducación en el patio de juegos. Barcelona: Universidad Autónoma de Barcelona - ICE.

BONILLA, A. y MARTÍNEZ, I. (1992). "Análisis del curriculum oculto de los modelos sexistas" en MORENO. Del silencio a la palabra. Serie de estudios No 32 , Madrid: Ministerio de Asuntos Sociales - Instituto de la Mujer, pp. 60-92.

BORES, N. y Otros (1994). "Análisis de las concepciones de educación física como instrumento para transformar la práctica docente" En ROMERO, Santiago (1994) Didáctica de la EF: diseños curriculares en primaria. Sevilla: Wanceulen. 
DELGADO, M. A., MEDINA, J. y VICIANA, J. (1996). "Evolution of implied theories about teaching in physical education teacher's preservice formation during the practice of teaching" Congress ICHPER-SD Europe. Coimbra.

DELGADO, M. A. y MEDINA, J. (1997). Investigación sobre las ciencias de la Actividad Física y del Deporte en la Universidad Española 1981-1996. Motricidad Humana. Revista Euro-Americana de las ciencias de la actividad física y el deporte. Vol. III. 1997 pp. 133150.

DELGADO, M. A. y Otros (1999). Investigaciones en la práctica de la enseñanza de la Educación Física. Granada: Universidad de Granada.

DELGADO, M. A. (2002). La investigación didáctica: De las teorías implícitas a la praxis de la educación física. II Congreso de Ciencias del Deporte. INEF de Madrid. Del 14 al 16 de Marzo de 2002. Madrid.

DELGADO, M. A., MEDINA, J. y CHILLÓN, P. (2002). "Cuestionario sobre las teorías implícitas de la educación física. ¿Qué opina el alumnado sobre al Educación Física?". Revista digital . www.efdeportes.com Buenos Aires, Año 8. N 50, julio de 2002.

DELGADO, M. A., MEDINA, J. y SOLANA, A. (2002). "Las teorías implícitas acerca de la EF en función del género" Congreso internacional de la AIESEP. A Coruña, 22 - 25 de octubre de 2002

DELGADO, M. A. y ZURITA. F. (2002). "Estudio de las teorías implícitas de la educación física en la formación inicial de los maestros en las diferentes especialidades. ¿Qué opinan los futuros maestros?". CRONOS. Revista universitaria de la actividad y el deporte. № 2 . Julio / Diciembre 2002. Páginas 34 - 44.

FERNÁNDEZ, E. (1995). Actividad Física y Género: Representaciones diferenciadas en el futuro profesor de Educación Primaria. Madrid: UNED.

GADNER, H. (1993). La mente no escolarizada. ¿Cómo piensan los niños y cómo deberían enseñar las escuelas? Buenos Aires: Paidós.

GEORGE, L. y KIRK, D. (1988). "Limits of change in physical education: ideologies, teachers and experience of physical activity". En EVANS, J. (Eds.) Teachers, teaching and control y Physical Education. The Falmer Press.

GERVILLA, E. (2000). Valores del cuerpo educando. Barcelona: Herder.

GRANLEESE, J.; TURNER, I.; TREW, K. (1989). "Teachers' and boys' anda girls' perception of competence in the primary school: The importance of physical competence" British journal of edcuational psycology, 59, (P+ 1), Feb 1989, 31 - 37.

GRIFFIN, P. S. (1985). "Boys' participation styles in a middle school Physical Education team sports unit". Journal of teaching in physical education., 4 (2), 100 110.
MCBRIDE, R. E. (1990). "Sex - Role Stereotyping behaviors among Elemental, Junior and Senior High School Physical Education Specialists" Journal of teaching in Physical Education, 9, 249 - 261.

MARTÍNEZ, L. (1994). "Influencia de las creencias implícitas en la formación inicial de los especialistas en EF" En ROMERO, Santiago (1994). Didáctica de la EF: diseños curriculares en primaria. Sevilla: Wanceulen.

MEDINA, J. y DELGADO, M. A. (1998). "Una primera aproximación a la investigación sobre las teorías del alumno acerca de la Educación Física". En García, A., Ruiz, F. Y Casimiro, A. J. (1998) Enseñanza de la Educación Física y el Deporte escolar. Almería: IAD.

MARRERO, J. (1993). "Diseño de la metodología para el estudio de las teorías implícitas". En RODRIGUEZ, A., RODRIGO, Ma José y MARRERO, J. (1993). Teorías implícitas. Una aproximación al conocimiento cotidiano. Madrid: Visor.

RODRÍGUEZ, A., RODRIGO, Ma José y MARRERO, $\mathrm{J}$. (1993). Teorías implícitas. Una aproximación al conocimiento cotidiano. Madrid: Visor.

SÁNCHEZ, F. (1996) La actividad física orientada hacia la salud. Madrid: Biblioteca Nueva.

SICILIA, A. (1997). Evolución del conocimiento escolar del estudiante de Bachiller en Educación Física, durante una actuación docente orientada hacia la autonomía de la enseñanza. Un estudio de casos. Tesis doctoral inédita. Universidad de Granada.

SICILIA, A. (1997b). "El alumno: objeto de estudio por parte del profesor" En Delgado, M. A. (1997) Formación y actualización del profesorado de EF y del entrenador deportivo. Experiencias en formación inicialy permanente. Sevilla: Wanceulem.

SUBIRATS, M. y TOME, A. (1992). "Pautas de observación para el análisis del sexismo en el ámbito educativo". Cuadernos de Coeducación. No 2. Barcelona: Instituto de Ciencias de la Educación - Universidad Autónoma de Barcelona.

STOLL, L y FINK, Dean (1999). Para cambiar nuestras escuelas. Reunir la eficacia y la mejora. Barcelona: Octaedro.

TEMPLIN, T.J. and OLSON, J.K. (1983). Teaching in Physical Education. Champaign. Illinois: Human Kinetics.

VÁZQUEZ, B. y ÁLVAREZ, G. (1990). Guía para una educación Física no sexista. Madrid: Ministerio de Educación y Ciencia.

VÁZQUEZ, B.; FERNÁNDEZ, E. et alii, (2000) Educación Física y Género. Modelo para la observación y el análisis del comportamiento del alumnado y del profesorado. Madrid: Gymnos.

WITTROCK, M. (1989). La investigación en la Enseñanza. Buenos Aires: Paidós. 


\section{ANEXOS}

\section{Anexo 1:}

CUESTIONARIO SOBRE LA EDUCACIÓN FÍSICA. PROFESORADO. (TIEFP).

Estudios:

Edad:
Especialidad:

Sexo:
Curso:

Fecha:

Estamos estudiando las concepciones de los futuros profesores y profesoras acerca de la Educación Física. A continuación se presenta una serie de frases (enunciados) en las que se recogen ideas y formas de entender la Educación Física. Queremos que nos indique en qué medida estas ideas se ajustan a las suyas. Gracias por su colaboración.

\section{Instrucciones del cuestionario:}

a) Lea atentamente las frases en las que se opina sobre la manera de entender la Educación Física.

b) Puntuará como 7 aquellas frases que correspondan fielmente a su idea sobre la Educación Física.

c) Puntuará como 0 aquellas frases que no correspondan en absoluto con su idea sobre la Educación Física.

d) Dará puntuaciones intermedias 2, 3, 4 y 5 a aquellas en las que la correspondencia no sea extrema.

e) En esta tarea no existen aciertos ni errores. Tenga en cuenta que se trata de dar puntuaciones que reflejen su opinión sobre el grado de relación de cada frase con las ideas que usted tiene acerca de la Educación Física.

Procure utilizar toda la escala de puntuaciones. Tómese el tiempo que considere necesario. Si ha comprendido la forma de rellenar este cuestionario, puede empezar.

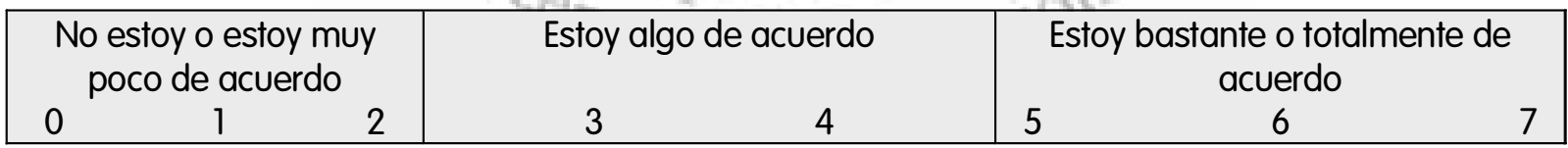

\begin{tabular}{|c|c|c|c|c|c|c|c|c|c|}
\hline \multicolumn{2}{|c|}{ ECUESTIONARIO NORMATIVO } & \multirow{2}{*}{\multicolumn{2}{|c|}{0 : }} & \multirow{3}{*}{2} & \multirow{3}{*}{\multicolumn{2}{|c|}{\begin{tabular}{l|l}
3 & 4 \\
\end{tabular}}} & \multirow{3}{*}{5} & & \\
\hline Ent & ciados & & & & & & & & 7 \\
\hline 1 & $\begin{array}{l}\text { Soy de los que opinan que los ejercicios físicos realizados en clase } \\
\text { de EF ayudan a nuestros alumnos /as para estar más sanos. }\end{array}$ & & & & & & & & \\
\hline 2 & Para mí lo más importante de la clase de EF son los ejercicios gimnásticos. & & & & & & & & \\
\hline 3 & Creo que en la EF jugar es esencial. & & & & & & & & \\
\hline 4 & Considero que en la EF se deben dar clases de danza. & & & & & & & & \\
\hline 5 & $\begin{array}{l}\text { Opino que la EF tiene valores formativos de la persona, como por } \\
\text { ejemplo, la voluntad y el compañerismo. }\end{array}$ & & & & & & & & \\
\hline 6 & La cultura física es tan importante como la cultura intelectual. & & & & & & & & \\
\hline 7 & $\begin{array}{l}\text { En mi opinión lo fundamental de la EF es la adquisición de hábitos de } \\
\text { ejercicio físico y salud. }\end{array}$ & & & & & & & & \\
\hline 8 & $\begin{array}{l}\text { Se debería insistir en la EF que lo más importante es aprender a } \\
\text { relacionarse con los demás. }\end{array}$ & & & & & & & & \\
\hline 9 & Creo que lo importante de la EF es la filosofía de la vida que enseña. & & & & & & & & \\
\hline 10 & $\begin{array}{l}\text { El médico debería plantear ejercicios de rehabilitación para que en } \\
\text { la EF los realizaran alumnos con lesiones temporales (exentos } \\
\text { temporales) y exentos totales. }\end{array}$ & & & & & & & & \\
\hline 11 & Creo que con la EF se puede mejorar el futuro de nuestro deporte de élite. & & & & & & & & \\
\hline 12 & LaEF permite jugar y a través del juego educar motrizmente. & & & & & & & & \\
\hline
\end{tabular}




\section{CUESTIONARIO NORMATIVO}

Enunciados

13 En la EF hay que fomentar el "fair play" en el deporte como una manera de afrontar la vida.

$14 \quad$ Se debería insistir más en las clases de EF cuestiones relacionadas con el ritmo y la música.

15 Pienso que lo más importante de la EF es el beneficio que proporciona a la salud.

16 Considero que en la EF con el poco tiempo que se dispone es preferible que los chicos se lo pasen bien.

17 Pienso que lo más importante de la EF es el beneficio que proporciona al desarrollo muscular.

18 Se debería insistir en la EF sobre la realización adecuada de los ejercicios para evitar lesiones.

19 Considero que en la EF se deben hacer ejercicios de mejora postural.

20 Opino que la EF sirve para desarrollar integralmente a la persona.

21 Pienso que lo que más demandan los alumnos en la EF es la educación expresiva.

22 En mi opinión lo importante de la EF es el disfrute de realizar actividad física, más que el rendimiento y el propio aprendizaje.

23 Considero que mejorar la ejecución técnica constituye la base del deporte a realizar en la clase de EF.

24 Opino que la EF sirve para compensar la falta de actividad física diaria del alumnado.

25 En EF cuando se hace deporte se está aprendiendo a que el alumnado se conozca mejor y a respetar a los demás.

26 Pienso que la Educación Física sirve para mantener la figura.

27 Creo que en la EF hay que aprender nociones de alimentación.

28 Creo que como mejor puedo enganchar a los alumnos a la EF es que se diviertan en las clases.

29 Soy de los que opinan que en las clases de EF hay que pasárselo estupendamente.

30 Opino que la EF pone "cachas" a los alumnos /as.

31 Considero que la EF desarrolla todos los aspectos de la persona.

32 En la EF lo ideal es que después de la clase se pudieran duchar los alumnos /as.

33 En la EF más que el deporte y la condición física, que es selectiva, se debe realizar expresión corporal.

34 En mi opinión la EF sólo es una asignatura en el curriculum escolar.

35 Soy de los que opinan que la EF permite expresarse a través del cuerpo.

36 En la clase de EF se pretende lograr más desarrollo muscular y corporal.

37 Creo que si sólo se realiza actividad física en la clase de EF los beneficios para la salud son escasos.

38 Estoy convencido del valor educativo creciente de la EF en nuestra sociedad.

39 Hacer EF permite realizar mejor algunas tareas de la vida diaria del alumnado.

$40 \quad$ Los beneficios de la expresión corporal en la EF no son sólo físicos sino sociales y de relación personal.

41 Opino que la EF sirve, por medio del juego, para desarrollar la personalidad del niño.

42 En la EF se aprende a mejorar las cualidades físicas.

43 En mi opinión en la EF es fundamental la comunicación no verbal (la expresión). 


\begin{tabular}{|c|c|c|c|c|c|c|c|c|c|}
\hline \multicolumn{10}{|c|}{ CUESTIONARIO NORMATIVO } \\
\hline \multicolumn{2}{|c|}{ Enunciados } & \multirow{2}{*}{\multicolumn{2}{|c|}{01}} & \multirow[t]{2}{*}{12} & \multirow{2}{*}{\multicolumn{2}{|c|}{\begin{tabular}{l|l}
3 & 4 \\
\end{tabular}}} & \multirow{2}{*}{\multicolumn{2}{|c|}{6}} & \multirow[t]{2}{*}{7} \\
\hline 44 & En la EF, sobre todo, se debería hacer deporte que es lo que más gusta al alumnado. & & & & & & & & \\
\hline 45 & Lo importante en la EF es el proceso de aprender no el resultado. & & & & & & & & \\
\hline 46 & $\begin{array}{l}\text { Considero que la EF sirve al alumnado para liberarse y olvidarse un rato } \\
\text { de los problemas diarios de las clases y para ello tiene que ser } \\
\text { entretenida. }\end{array}$ & & & & & & & & \\
\hline 47 & $\begin{array}{l}\text { Creo que en la EF hay que aprender a comunicarse motrizmente como } \\
\text { experiencia importante para el individuo. }\end{array}$ & & & & & & & & \\
\hline 48 & $\begin{array}{l}\text { La EF sirve para quitarse los kilos de más que se cogen y para estar en } \\
\text { forma. }\end{array}$ & & & & & & & & \\
\hline 49 & Pienso que lo más importante de la EF es lo vivencial y lo lúdico. & & & & & & & & \\
\hline 50 & Opino que la EF ayuda a través de la expresión corporal a desinhibirse. & & & & & & & & \\
\hline & & 0 & 1 & 2 & 3 & 4 & $5 \mid 6$ & 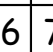 & \\
\hline
\end{tabular}

